\title{
Management of Feline Idiopathic Hypertension with Target Organ Damage: A Case Report
}

\section{Ji-Hye Lee \\ Jihee Kim \\ Yoonji Kim \\ Soomin Kim \\ Hyeon-Jin Kim \\ Ha-Jung Kim*}

Department of Veterinary Internal Medicine, College of Veterinary Medicine, Chonnam National University, Gwangju 61186, Korea
*Correspondence: kimhj614@jnu.ac.kr

\section{ORCID}

Ji-Hye Lee:

https://orcid.org/0000-0002-1548-6598

Jihee Kim:

https://orcid.org/0000-0003-0885-8784

Yoonji Kim:

https://orcid.org/0000-0003-0690-2745

Soomin Kim:

https://orcid.org/0000-0002-9419-1412

Hyeon-Jin Kim:

https://orcid.org/0000-0003-2751-2201

Ha-Jung Kim:

https://orcid.org/0000-0002-2699-0263

Copyright $\odot$ The Korean Society of Veterinary Clinics
Abstract An 11-years old spayed female Persian chinchilla cat presented with acute onset of blindness from bilateral retinal detachment and systemic hypertension. On physical examination, the cat was tachycardic ( 240 beats $/ \mathrm{min}$ ) with a systolic blood pressure of around $250 \mathrm{mmHg}$. Clinical findings, blood works, urinalysis, thyroid function test, radiography, echocardiogram, and ultrasonography were performed to rule out underlying diseases. Organ injury induced by systemic hypertension including bilateral retinal detachment and left ventricular hypertrophy were confirmed by ultrasound. Based on these results, it was diagnosed as feline idiopathic hypertension with target organ damage (TOD). The cat was treated with a combination therapy using high doses of amlodipine, telmisartan, and atenolol. After the treatment, its hypertension and TOD were improved. This case showed that appropriate therapeutic management can help prevent TOD associated with feline hypertension.

Key words calcium channel blocker, angiotensin-converting-enzyme inhibitor, angiotensin II receptor blocker, systemic hypertension, cat. 


\section{Introduction}

Systemic hypertension is a common disease in older cats. It can be classified as idiopathic (or primary) hypertension and secondary hypertension. Secondary hypertension may be seen with many diseases including chronic kidney disease (CKD), hyperthyroidism, primary hyperaldosteronism (PHA), hyperadrenocorticism (HAC), and pheochromocytoma (14). Idiopathic hypertension refers to persistent pathological hypertension in the absence of an overt clinically apparent disease known to cause secondary hypertension (1). Although secondary hypertension is most common in cats, idiopathic hypertension is more common than previously recognized, accounting for approximately $13-20 \%$ of cases in cats (10).

Sustained high blood pressure can damage tissues. Such damage is commonly referred to as target organ damage (TOD). Hypertension is most likely to cause diseases in kidneys, eyes, brain, heart, and blood vessels that are rich in arterial and arteriolar supply as a result of increased systemic vascular resistance (14). TOD is associated with considerable morbidities, including blindness, ventricular hypertrophy, proteinuria, and hypertensive encephalopathy (4).

Ocular signs are the most common and specific symptoms associated with the diagnosis of hypertension (3). Cats with hypertensive ocular diseases most commonly present with partial or complete bullous retinal detachment. Subretinal edema, subretinal or intraretinal hemorrhage, retinal tears, retinal disinsertion, and hyphema can also develop (16).

Feline hypertension associated with clinical cardiac signs such as heart murmur, dyspnea, and gallop rhythm has been frequently reported. In response to chronic pressure overload, left ventricular wall thickness would increase proportionally to increase of blood pressure to maintain normal wall stress (3). Echocardiography may show hypertrophies of the ventricular septum and ventricular posterior wall, left atrial enlargement, and an abnormal mitral inflow pattern (15).

The purpose of antihypertensive treatment is to effectively control systolic blood pressure (SBP) as quickly as possible in order to decrease the risk of further TOD (2). Consequently, a combination of early recognition and initiation of appropriate treatments can reduce morbidities associated with hypertension (8). The present study reports a case of severe idiopathic hypertension with TOD in a cat that is successfully managed.

\section{Case Report}

An 11-years old, spayed female, Persian chinchilla cat presented with severe systemic hypertension and an acute onset of blindness resulting from retinal detachment. The owner reported that the cat had no reaction to movement and that it had been bumping into stationary objects. It had no history of trauma or exposure to toxic agents. On physical examination, the cat was tachycardic ( 240 beats/min) with a systolic blood pressure of around $250 \mathrm{mmHg}$ measured with an ultrasonic Doppler flow detector. Its respiratory sounds and rectal temperature were normal. No heart murmur was noted.

Examination of its eyes with a direct ophthalmoscope, slit lamp examination, and ultrasonography revealed dilated pupils, absent direct and consensual pupillary light reflexes, hyphema (Fig. 1A), retinal hemorrhages, and bilateral retinal detachment (Fig. 1B). Its menace response was absent bilaterally. Its blindness was attributed to bilateral retinal detachment.

Complete blood count (CBC), serum biochemistry, blood electrolytes, and urinalysis were performed, showing no remarkable findings. Its serum symmetric dimethylarginine (SDMA) concentrations were within normal limits $(14 \mathrm{ug} / \mathrm{dL}$; reference range, 0-18 ug/dL). Renal ultrasound examination showed a number of urinary stones in bilateral renal diverticulum and ureter that did not cause obstruction.

Total thyroxine (T4) and free T4 were measured to assess its thyroid function. They were within normal reference ranges (Total T4: $1.5 \mathrm{ug} / \mathrm{dL}$, reference range: $0.8-4.7 \mathrm{ug} / \mathrm{dL}$; free $\mathrm{T4}$ : $1.7 \mathrm{ng} / \mathrm{dL}$, reference range: $0.7-2.6 \mathrm{ng} / \mathrm{dL}$ ). There was nothing unusual when thyroid glands were observed using radiogra-

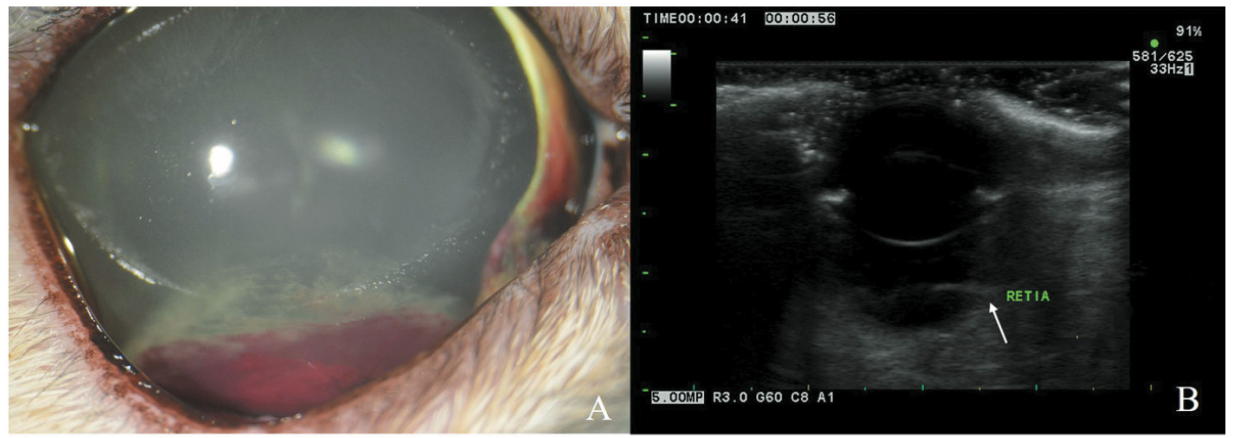

Fig. 1. Photographs of an 11-year-old cat with sudden onset blindness with a presumptive diagnosis of hypertensive retinopathy. (A) Slit lamp examination revealing hyphema associated with an increased intraocular pressure. (B) Ophthalmic ultrasonography showing that the retina is detached from the eyeball wall (arrow). 


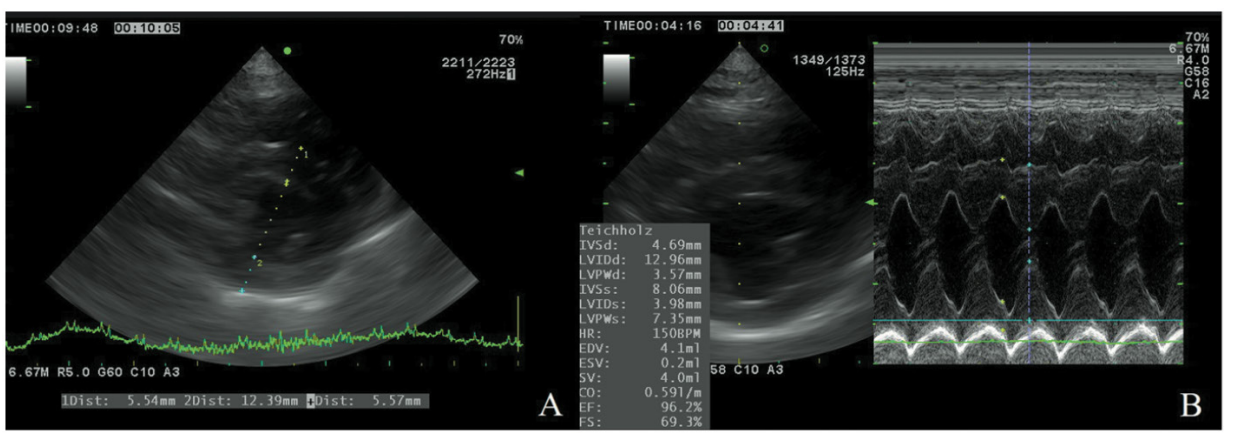

Fig. 2. Echocardiogram of an 11-year-old cat with systemic hypertensive injury acquired from the right parasternal short-axis view. (A) Echocardiogram revealing IVS (5.5 $\mathrm{mm}$, ref. range 2.2-4 $\mathrm{mm}$ ) and LVPW (5.5 $\mathrm{mm}$, ref. range 2.2-4.4 $\mathrm{mm}$ ) at the time of diagnosis. (B) Echocardiogram revealing IVS $4.6 \mathrm{~mm}$ and LVPW $3.5 \mathrm{~mm}$ after antihypertensive treatment.

phy and ultrasonography. Additionally, no remarkable findings were detected in ultrasonography of adrenal glands.

Echocardiogram revealed mild increase of interventricular septum (IVS) (5.5 mm, reference range: $2.2-4 \mathrm{~mm}$ ) and left ventricular posterior wall (LVPW) $(5.5 \mathrm{~mm}$, reference range: 2.2-4.4 mm) at end diastole (Fig. 2A). On electrocardiogram, sinus tachycardia, complete left bundle branch block, and left atrial enlargement were detected. These were considered to be due to systemic hypertensive injury that caused secondary changes on the heart regardless of primary hypertrophic cardiomyopathy (HCM). Based on these diagnostic tests, the case was diagnosed as feline idiopathic hypertension.

Treatment was started by administering amlodipine (0.625 $\mathrm{mg} / \mathrm{cat}, \mathrm{PO}$ sid, JW Pharmaceutical Corp., Danjin, Korea) and telmisartan $(1.5 \mathrm{mg} / \mathrm{kg}, \mathrm{PO}$ sid, Boehringer Ingelheim Promeco, Barrio Xaltocan, Mexico). Additionally, atenolol $(6.25 \mathrm{mg} /$ cat, PO bid, KyungDong pharmaceutical Corp., Hwaseong, Korea) was administered orally to decrease its heart rate and heart contractibility. Blood pressure readings were taken one week later, showing no notable decrease in systolic blood pressure (SBP). Therefore, the dose of amlodipinea was doubled (1.25 mg/cat, PO sid, JW Pharmaceutical Corp., Danjin, Korea). After it was taken for a week, its SBP was decreased to be within normal levels.

After a month of antihypertensive treatment, interventricular septum and left ventricular posterior wall at end diastole decreased by about 27\% (Fig. 2B). Although the antihypertensive therapy was continued for two months, its eyes with retinal detachment and a menace response were not recovered. The patient has reached target SBP $(<160 \mathrm{mmHg})$ which has been maintained for two months.

\section{Discussion}

This study describes a clinical case of feline idiopathic hypertension treated with combination drugs of amlodipine, telmisartan, and atenolol. At the time of diagnosis, its ophthalmic and cardiovascular abnormalities were evidenced by blindness, secondary HCM, and additional electrocardial imbalances as TOD appearance. According to one study, the vast majority (87\%) of hypertensive cats have either cardiac or ocular abnormalities at the time of first diagnosis (6). Most cats with blood pressure $\geq 180 \mathrm{mmHg}$ at diagnosis show clinical signs of TOD at diagnosis (4). In initially normotensive cats having regular blood pressure monitoring, $52 \%$ of them have evidence of TOD at the point when they are diagnosed with hypertension, lower than the proportion of cats with TOD at time of diagnosis with severe hypertension (4).

TOD is more associated with survival time than blood pressure at diagnosis, suggesting that the severity of hypertension is associated with survival after diagnosis rather than the extent of hypertension (4). There is an association between urine protein to creatinine ratio (UPC) and survival after hypertension diagnosis (4). Median survival time has been previously estimated to be up to 490 days for cats that are not proteinuric at diagnosis, whereas the median survival time has been estimated to be 162 days for cats that are proteinuric at diagnosis (4). Additionally, retinal detachment and tortuous vessels identified at diagnosis of hypertension are associated with increased morbidities (4).

Amlodipine besylate is currently regarded as the primary antihypertensive treatment of choice in cats. It acts by blocking L-type calcium channels in vascular smooth muscle and produces its antihypertensive effects by reducing peripheral resistance (2). In spite of administration of amlodipine besylate at a lower dose $(0.625 \mathrm{mg} / \mathrm{cat}, \mathrm{PO}$ sid, JW Pharmaceutical Corp., Danjin, Korea), the cat had no significant change in blood pressure. Blood pressure dropped to target SBP $(<160 \mathrm{mmHg})$ after administration of amlodipine besylate at a higher dose $(1.25 \mathrm{mg} / \mathrm{cat}, \mathrm{PO}$ sid, JW Pharmaceutical Corp., Danjin, Korea). It has been suggested that cats with SBP > $200 \mathrm{mmHg}$ may benefit from a higher starting dosage (1.25 mg per cat per day) of amlodipine besylate (1).

Telmisartan is an angiotensin receptor blocker (ARB) that can selectively block angiotensin-II type 1 receptor (AT1 receptor) with a high affinity and leave angiotensin-II type 2 
receptor (AT2 receptor) available for activation (13). Thus, it can inhibit pro-hypertensive effects including systemic vasoconstriction, intravascular fluid expansion, and sympathetic activation (8). AT2 receptor mediates beneficial actions of angiotensin-II (AT-II) such as vasodilation and natriuresis (11). Telmisartan can dilate the renal efferent arteriole, thereby decreasing intraglomerular pressure. It has been proven to be an effective antiproteinuric drug $(1,8)$. The combination of amlodipine besylate and telmisartan is expected to have antiproteinuric efficacy. There was no progression of proteinuria in the cat of the present case.

Atenolol can be introduced into cats with amlodipine treatment in an attempt to improve control of systolic arterial blood pressure where heart rate is high or cardiac arrhythmia is detected (6). Beta-blockers, particularly atenolol, may be useful for controlling heart rates in some tachycardic hypertensive cats (9). However, antihypertensive effects of monotherapy with atenolol have not been reported yet.

Following an antihypertensive therapy, eyes with retinal detachment have reattached (69\%) and eyes with negative menace responses at presentation have recovered a menace response (57.6\%) (16). Eyes with a complete retinal detachment for greater than two weeks are less likely to regain vision because photoreceptor synaptic terminals undergo marked and rapid degeneration between 13 days and 30 days following detachment (7). The cat in the present case did not recovered a menace response. It did not restore vision during the monitoring period after treatment for systemic hypertension. One explanation for these findings was that the original episode of retinal detachment which required some time to become evident might have led to retinal degeneration so that there were no changes in visual outcome after treatment. Another explanation was that severe hypertension might have resulted in irreversible ischemic damage to the choriocapillaris, resulting in progression of retinal degeneration even though hypertension was controlled (16).

Left ventricular mass and wall thickness can gradually increase in proportion to total peripheral resistance and arterial pressure in human with essential hypertension (5). Since left ventricular hypertrophy is associated with increased morbidity and mortality in man (12), evaluating blood pressure in cats with left ventricular hypertrophy might be clinically relevant. Effective antihypertensive treatment may decrease the prevalence of left ventricular hypertrophy (LVH) in affected cats, thus reducing morbidity and mortality in cats.

This case report has some limitations. First, aldosterone and cortisol concentrations were not measured for this cat. Its diagnosis was made only through clinical signs, electrolytes testing, and adrenal ultrasonography. There is further limitation in that menace response was used as a measure of vision in this cat. The presence of menace is a too crude test to differentiate subtle differences in vision (16).

\section{Conclusions}

This case showed that a combination therapy using higher doses of a calcium channel blocker (CCB), an ARB, and a beta blocker was effective in lowering blood pressure and reducing heart rate of a cat with feline idiopathic hypertension. This antihypertensive treatment decreased the risk of progressive TOD and prevented concentric left ventricular hypertrophy. Therefore, severe complications of heart were not reported in this affected cat.

\section{Acknowledgements}

We thank Chonnam Veterinary Teaching Hospital for its support and the dog's owner.

\section{Conflicts of Interest}

The authors have no conflicting interests.

\section{Source of Funding}

This research was supported by the Basic Science Research Program through the National Research Foundation of Korea (NRF), funded by the Ministry of Education (NRF2020R1A2C2005364).

\section{References}

1. Acierno MJ, Brown S, Coleman AE, Jepson RE, Papich M, Stepien $R L$, et al. ACVIM consensus statement: guidelines for the identification, evaluation, and management of systemic hypertension in dogs and cats. J Vet Intern Med 2018; 32: 1803-1822

2. Bijsmans ES, Doig M, Jepson RE, Syme HM, Elliott J, Pelligand L. Factors influencing the relationship between the dose of amlodipine required for blood pressure control and change in blood pressure in hypertensive cats. J Vet Intern Med 2016; 30: 1630-1636.

3. Chetboul V, Lefebvre HP, Pinhas C, Clerc B, Boussouf M, PoucheIon JL. Spontaneous feline hypertension: clinical and echocardiographic abnormalities, and survival rate. J Vet Intern Med 2003; 17: 89-95.

4. Conroy M, Chang YM, Brodbelt D, Elliott J. Survival after diagnosis of hypertension in cats attending primary care practice in the United Kingdom. J Vet Intern Med 2018; 32: 1846-1855.

5. Dunn FG, Chandraratna P, deCarvalho JG, Basta LL, Frohlich ED. 
Pathophysiologic assessment of hypertensive heart disease with echocardiography. Am J Cardiol 1977; 39: 789-795.

6. Elliott J, Barber PJ, Syme HM, Rawlings JM, Markwell PJ. Feline hypertension: clinical findings and response to antihypertensive treatment in 30 cases. J Small Anim Pract 2001; 42: 122-129.

7. Erickson PA, Fisher SK, Anderson DH, Stern WH, Borgula GA. Retinal detachment in the cat: the outer nuclear and outer plexiform layers. Invest Ophthalmol Vis Sci 1983; 24: 927-942.

8. Glaus TM, Elliott J, Herberich E, Zimmering T, Albrecht B. Efficacy of long-term oral telmisartan treatment in cats with hypertension: results of a prospective European clinical trial. J Vet Intern Med 2019; 33: 413-422.

9. Henik RA, Stepien RL, Wenholz LJ, Dolson MK. Efficacy of atenolol as a single antihypertensive agent in hyperthyroid cats. J Feline Med Surg 2008; 10: 577-582.

10. Jepson RE. Feline systemic hypertension: classification and pathogenesis. J Feline Med Surg 2011; 13: 25-34.

11. Kemp BA, Howell NL, Keller SR, Gildea JJ, Padia SH, Carey RM.
AT2 receptor activation prevents sodium retention and reduces blood pressure in angiotensin II-dependent hypertension. Circ Res 2016; 119: 532-543.

12. Lesser M, Fox PR, Bond BR. Assessment of hypertension in 40 cats with left ventricular hypertrophy by Doppler-shift sphygmomanometry. J Small Anim Pract 1992; 33: 55-58.

13. Sent U, Gössl R, Elliott J, Syme HM, Zimmering T. Comparison of efficacy of long-term oral treatment with telmisartan and benazepril in cats with chronic kidney disease. J Vet Intern Med 2015; 29: 1479-1487.

14. Taylor SS, Sparkes AH, Briscoe K, Carter J, Sala SC, Jepson RE, et al. ISFM consensus guidelines on the diagnosis and management of hypertension in cats. J Feline Med Surg 2017; 19: 288-303.

15. Van Boxtel SA. Hypertensive retinopathy in a cat. Can Vet J 2003; 44: 147-149.

16. Young WM, Zheng C, Davidson MG, Westermeyer HD. Visual outcome in cats with hypertensive chorioretinopathy. Vet Ophthalmol 2019; 22: 161-167. 